\title{
Observer-based consensus of second-order multi-agent systems without velocity measurements
}

\author{
Chenglin Wen ${ }^{1}$, Fang Liu $^{2}$, Qiang Song ${ }^{1, *}$, Xiaoliang Feng ${ }^{1}$ \\ ${ }^{1}$ College of Electrical Engineering, Henan University of Technology, Zhengzhou 450001, China \\ ${ }^{2}$ School of Information Engineering, Huanghuai University, Zhumadian, Henan 463000, China
}

\begin{abstract}
This paper investigates the consensus of second-order multi-agent systems without measuring the velocity states of the agents, where each agent can be either a double integrator or a harmonic oscillator. By utilizing position information of the agents, a distributed observer-based protocol is proposed to solve the second-order consensus problem of multi-agent systems with or without time delay. The observer-based consensus problem is converted to the stability analysis for a set of second-order quasipolynomials through coordinate transform and system decomposition. Some necessary and sufficient conditions are derived for reaching second-order consensus in multi-agent systems with or without time delay, respectively, and it is shown that the consensus can be achieved if and only if the communication delay is less than a critical value. Simulation examples are given to verify the theoretical analysis.
\end{abstract}

Keywords: Second-order consensus; multi-agent system; distributed observer; double integrator; harmonic oscillator; time delay.

\section{Introduction}

In the past few decades, the consensus problem has been a focal point of many different disciplines due to its important role in the investigation of the distributed coordination of multi-agent systems. The consensus in a multi-agent system is said to be reached if all the agents can eventually agree on some common value under some control algorithms based on local information. Much progress has been made in the consensus of multi-agent systems with first-order dynamics [1,2,3], second-order dynamics $[4,5,6,7,8,9]$, higher-order dynamics $[10,11,12,13]$ and fractional-order dynamics $[14,15]$. In particular, much effort has been devoted to the second-order consensus problem due to many practical applications of second-order multi-agent systems, such as the flocking of unmanned air vehicles [16, 17] and the formation control of multiple mobile robots $[18,19]$. It is worth mentioning that the first-order consensus problem is closely related to the synchronization problem of complex networks [20, 21, 22, 23].

A linear second-order multi-agent system is composed of a set of interconnected systems, where each agent may be a double integrator $[4,5,6]$, a spring-mass system [24], or a harmonic oscillator

\footnotetext{
${ }^{*}$ Corresponding author: e-mail: qsongseu@gmail.com (Q. Song)
} 
$[25,26]$. Moreover, note that a harmonic oscillator and a double integrators can be described by a unified mathematical model. Some fundamental results have been obtained to address the second-order consensus of multi-agent systems with double-integrator dynamics $[4,5,6]$. In recent years, the consensus problem of coupled harmonic oscillators has been intensively investigated by using many different control strategies $[25,26,27,28,29]$. It is now well known that the existence of a directed spanning tree in network topology is a necessary condition for reaching second-order consensus [4], and both the real and imaginary parts of the nonzero eigenvalues of network Laplacian matrix are important to discuss the consensus problem [5].

Since the time delay is inevitable in many physical systems [30,31, 32], some researchers have studied the consensus in a network of double integrators or harmonic oscillators with time delay. For coupled double-integrators, Yu et al. [5] showed that the communication delay should be less than some critical value and Meng et al. [6] further analyzed the effects of input and communication delays on second-order consensus. As for coupled harmonic oscillators, Zhang et al. [27] considered the consensus in the network with delayed velocity coupling, while Song et al. [29] investigated the consensus using delayed position information and proved that the time delay should be chosen from a set of bounded intervals.

Note that most consensus protocols for coupled double integrators $[4,5,6]$ and coupled harmonic oscillators all require velocity states $[25,26,27,28]$. However, for a second-order multi-agent system without being equipped with velocity sensors, the velocity information will not be available. Moreover, in some practical cases, the velocity might be more difficult to be accurately measured compared with the position. In recent years, by using current position measurements, some distributed observer-based consensus protocols have been developed for coupled double integrators [33, 34, 35, 36] and coupled harmonic oscillators [37], where only the algorithm in [36] assumes general directed network topology. As far as we know, very few reduced-order observer-based consensus protocols have been designed for second-order multi-agent systems with directed topology and time delay.

Motivated by the above-mentioned discussions, this paper studies the observer-based consensus for a class of linear second-order multi-agent systems, where each agent can be a double integrator or a harmonic oscillator. The main contribution of this paper is two-fold. First, by utilizing position information of the agents, we propose a distributed observer approach to solve the second-order consensus problems for coupled harmonic oscillators or coupled double integrators under directed network topology with or without communication delay. Then, through coordinate transformation and system decomposition, the consensus problem is converted to the stability analysis for a set of a second-order quasi-polynomials, based on which some necessary and sufficient conditions are derived for reaching second-order consensus in multi-agent systems with or without time delay, respectively. Second, we analyze the effects of communication delay on second-order consensus of multi-agent systems under the proposed observer-based protocol: on the one hand, it is shown that the consensus can be reached only when the communication delay is less than some critical value; on the other hand, it is found that the final consensus state is only determined by network topology, initial state and a model parameter of multi-agent system, indicating that the final consensus state is independent of the communication delay.

The rest of this paper is organized as follows. Section 2 designs an observer-based consensus protocol for second-order multi-agent systems based on position measurements. In Section 3, the consensus 
problem is transformed and analyzed via coordinate transformation. Sections 4 and 5 provide some necessary and sufficient conditions for reaching second-order consensus in multi-agent systems without and with time delay, respectively. Numerical examples are given in Section 6 to verify the theoretical analysis. Finally, some concluding remarks and future trends are stated in Section 7.

Notation: Let $\mathbb{Z}^{+}, \mathbb{R}$ and $\mathbb{C}$ represent the sets of nonnegative integers, real numbers and complex numbers, respectively. For $z \in \mathbb{C}$, let $\bar{z}, \operatorname{Arg}(z),|z|, \operatorname{Re}(z)$ and $\operatorname{Im}(z)$ be its conjugate, principal argument, modulus, real part and imaginary part, respectively, where $-\pi<\operatorname{Arg}(z) \leq \pi$. Denote the imaginary unit by $\mathbf{i}=\sqrt{-1}$. Let $I_{n}$ be the $n$-dimensional identity matrix, $1_{n} \in \mathbb{R}^{n}\left(0_{n} \in \mathbb{R}^{n}\right)$ be the vector of all ones (zeros), and $O_{n}$ be the square matrix with all entries being zeros. A vector $X$ is called nonnegative and denoted as $X \geq 0$ if all its elements are nonnegative. The determinant of the square matrix $A$ is denoted by $\operatorname{det}(A)$. The traditional sign function is denoted by $\operatorname{sign}(\cdot)$.

\section{Observer-based consensus protocol for second-order multi-agent sys- tem utilizing position information}

In this section, a distributed reduced-order observer-based approach using position information is proposed to achieve second-order consensus in coupled harmonic oscillators or coupled double integrators.

Consider the following multi-agent system composed of $N$ identical agents with second-order dynamics

$$
\begin{aligned}
\dot{x}_{i}(t) & =v_{i}(t) \\
\dot{v}_{i}(t) & =-\alpha x_{i}(t)+u_{i}(t), \quad i=1, \ldots, N,
\end{aligned}
$$

where $\alpha \geq 0$ is a constant, $x_{i} \in \mathbb{R}^{n}$ and $v_{i} \in \mathbb{R}^{n}$ are the position and velocity states of the $i$ th agent, respectively, and $u_{i} \in \mathbb{R}^{n}$ is the control input to be designed to achieve consensus, that is, $\lim _{t \rightarrow \infty} \| x_{i}(t)-$ $x_{j}(t) \|=0$ and $\lim _{t \rightarrow \infty}\left\|v_{i}(t)-v_{j}(t)\right\|=0, \forall i, j=1, \ldots, N(i \neq j)$. For notational brevity, it is assumed $n=1$ in this paper. Nevertheless, by using the Kronecker product [38], the theoretical results can be easily extended to the higher-dimensional case with $n \geq 2$.

It should be noted that each agent in multi-agent system (1) is a double integrator $[4,5,6]$ and a harmonic oscillator [25, 26] when $\alpha=0$ and $\alpha>0$, respectively. Hence, a class of second-order multi-agent systems can be generalized by the network model (1).

Let $\mathcal{G}$ be the digraph describing the topology of network (1). Let $\mathcal{A}=\left(a_{i j}\right)_{N \times N}$ be the adjacency matrix associated with $\mathcal{G}$ whose off-diagonal elements are defined as follows: if there is a directed link from node $j$ to node $i(i \neq j)$, then $a_{i j}>0$; otherwise $a_{i j}=0$. In this paper, the graph $\mathcal{G}$ is assumed to be simple without self-loops, that is, $a_{i i}=0$ for all $i=1, \ldots, N$. On the basis of the adjacency matrix $\mathcal{A}$, the Laplacian matrix $L=\left(l_{i j}\right)_{N \times N}$ is given by: $l_{i j}=-a_{i j} \leq 0(i \neq j)$ and $l_{i i}=\sum_{j=1, j \neq i}^{N} a_{i j}[2,4,5]$.

In [11], Li et al. proposed a distributed reduced-order observer-based consensus protocol for delayfree multi-agent systems with general linear dynamics by using the outputs of the agents. Inspired by the work in [11] and the technique for Luenberger reduced-order observer [39], we design the following 
observer-based approach for reaching consensus in network (1) based on delayed position states

$$
\begin{aligned}
\dot{w}_{i}(t)= & -\alpha x_{i}(t)-x_{i}(t)-w_{i}(t)+u_{i}(t), \\
u_{i}(t)= & \left(k_{1}+k_{2}\right) \sum_{j=1, j \neq i}^{N} a_{i j}\left(x_{j}(t-\tau)-x_{i}(t-\tau)\right)+k_{2} \sum_{j=1, j \neq i}^{N} a_{i j}\left(w_{j}(t-\tau)-w_{i}(t-\tau)\right), \\
& i=1, \ldots, N,
\end{aligned}
$$

where $w_{i} \in \mathbb{R}$ is the state of the observer, $\tau \geq 0$ is the communication delay, $a_{i j}$ is the $(i, j)$-th element of the adjacency matrix $\mathcal{A}, k_{1}$ and $k_{2}$ are the coupling strengths to be designed.

Remark 1 For each agent in network (1), letting $z_{i}=\left(x_{i}, v_{i}\right)^{T}$, one has $\dot{z}_{i}=A z_{i}+B u_{i}, y_{i}=C z_{i}, i=$ $1, \ldots, N$, where $A=\left(\begin{array}{cc}0 & 1 \\ -\alpha & 0\end{array}\right), B=\left(\begin{array}{l}0 \\ 1\end{array}\right)$ and $C=\left(\begin{array}{ll}1 & 0\end{array}\right)$. It is easy to verify that the matrix pair $(A, B, C)$ is controllable and observable. In Ref. [39], some techniques have been proposed to design reduced-order observers for linear time-invariant systems. Considering the formula (3.5) in Ref. [39] and the structure of matrices $A, B$ and $C$, let $A_{11}=0, A_{12}=1, A_{21}=-\alpha, A_{22}=0, B_{1}=0$ and $B_{2}=1$. Then one can develop observer-based protocol (2) on the basis of the delayed position information.

Remark 2 Note that algorithm (2) is a kind of reduced-order observer-based protocol for multi-agent system (1) based on position states of the agents, and provides a unified approach to solve the second-order consensus problem for multi-agent systems composed of either double integrators or harmonic oscillators. In particular, the traditional separation principle in control theory still holds for the observer-based protocol (2), which will be further discussed in Remark 5.

Let $x(t)=\left(x_{1}(t), \ldots, x_{N}(t)\right)^{T}, v(t)=\left(v_{1}(t), \ldots, v_{N}(t)\right)^{T}$ and $w(t)=\left(w_{1}(t), \ldots, w_{N}(t)\right)^{T}$. By the definition of Laplacian matrix, the closed-loop network with dynamics (1) and (2) can be written as

$$
\begin{aligned}
\dot{x}(t) & =v(t) \\
\dot{v}(t) & =-\alpha x(t)-\left(k_{1}+k_{2}\right) L x(t-\tau)-k_{2} L w(t-\tau) \\
\dot{w}(t) & =-(\alpha+1) x(t)-w(t)-\left(k_{1}+k_{2}\right) L x(t-\tau)-k_{2} L w(t-\tau) .
\end{aligned}
$$

\section{Problem transformation and analysis}

This section analyzes the consensus problem for multi-agent system (1) under observer-based protocol (2) by using coordinate transformation and system decomposition. In particular, the final consensus state is explicitly determined.

The following lemma is important to derive the main results of this paper.

Lemma 1 [2] The Laplacian matrix L has a simple zero eigenvalue and all the other $N-1$ eigenvalues have positive real parts if and only if the digraph $\mathcal{G}$ contains a directed spanning tree, that is, there exists at least one node having a directed path to every other node. Moreover, $1_{N}$ and $\xi$ are the right and left eigenvectors associated with the simple zero eigenvalue of $L$, respectively, where $\xi=\left(\xi_{1}, \ldots, \xi_{N}\right)^{T}$ is nonnegative satisfying $\xi^{T} L=0$ and $\xi^{T} 1_{N}=1$. 
For network (3), assume that $\mathcal{G}$ contains a directed spanning tree. By Lemma 1, we know that the Laplacian matrix $L$ has a simple zero eigenvalue, whose corresponding right and left eigenvectors are $1_{N}$ and $\xi=\left(\xi_{1}, \ldots, \xi_{N}\right)^{T}$, respectively. Then, for Laplacian matrix $L$, there should exist nonsingular matrices $P=\left(p_{1}, \ldots, p_{N}\right) \in \mathbb{R}^{N \times N}$ and $P^{-1}=\left(q_{1}, \ldots, q_{N}\right)^{T} \in \mathbb{R}^{N \times N}$, in which one can let $p_{1}=1_{N}$ and $q_{1}=\xi[4]$, such that

$$
J=P^{-1} L P=\left(\begin{array}{cc}
0 & 0_{N-1}^{T} \\
0_{N-1} & \widetilde{L}
\end{array}\right),
$$

where $J$ is the Jordan canonical form of $L$ and the diagonal elements of $\widetilde{L}$ are nonzero eigenvalues of $L$.

For network (3), consider the following coordinate transformation

$$
\widetilde{x}(t)=P^{-1} x(t), \widetilde{v}(t)=P^{-1} v(t), \widetilde{w}(t)=P^{-1} w(t),
$$

where $\widetilde{x}(t)=\left(\widetilde{x}_{1}(t), \ldots, \widetilde{x}_{N}(t)\right)^{T}, \widetilde{v}(t)=\left(\widetilde{v}_{1}(t), \ldots, \widetilde{v}_{N}(t)\right)^{T}$ and $\widetilde{w}(t)=\left(\widetilde{w}_{1}(t), \ldots, \widetilde{w}_{N}(t)\right)^{T}$.

It follows from (3) and (5) that

$$
\begin{aligned}
\dot{\widetilde{x}}(t) & =\widetilde{v}(t), \\
\dot{\widetilde{v}}(t) & =-\alpha \widetilde{x}(t)-\left(k_{1}+k_{2}\right) P^{-1} L P \widetilde{x}(t-\tau)-k_{2} P^{-1} L P \widetilde{w}(t-\tau), \\
\dot{\widetilde{w}}(t) & =-(\alpha+1) \widetilde{x}(t)-\widetilde{w}(t)-\left(k_{1}+k_{2}\right) P^{-1} L P \widetilde{x}(t-\tau)-k_{2} P^{-1} L P \widetilde{w}(t-\tau) .
\end{aligned}
$$

By virtue of (4), system (6) can be decomposed into two subsystems as follows

$$
\begin{aligned}
& \dot{\widetilde{x}}_{1}(t)=\widetilde{v}_{1}(t) \\
& \dot{\widetilde{v}}_{1}(t)=-\alpha \widetilde{x}_{1}(t) \\
& \dot{\widetilde{w}}_{1}(t)=-(\alpha+1) \widetilde{x}_{1}(t)-\widetilde{w}_{1}(t) .
\end{aligned}
$$

and

$$
\begin{aligned}
& \dot{\dot{x}}(t)=\breve{v}(t) \\
& \dot{\vec{v}}(t)=-\alpha \breve{x}(t)-\left(k_{1}+k_{2}\right) \widetilde{L} \breve{x}(t-\tau)-k_{2} \widetilde{L} \breve{w}(t-\tau) \\
& \dot{\dot{w}}(t)=-(\alpha+1) \breve{x}(t)-\breve{w}(t)-\left(k_{1}+k_{2}\right) \widetilde{L} \breve{x}(t-\tau)-k_{2} \widetilde{L} \breve{w}(t-\tau),
\end{aligned}
$$

where $\breve{x}(t)=\left(\widetilde{x}_{2}(t), \ldots, \widetilde{x}_{N}(t)\right)^{T}, \breve{v}(t)=\left(\widetilde{v}_{2}(t), \ldots, \widetilde{v}_{N}(t)\right)^{T}$ and $\breve{w}(t)=\left(\widetilde{w}_{2}(t), \ldots, \widetilde{w}_{N}(t)\right)^{T}$.

Let $y(t)=\left(\breve{x}^{T}(t), \breve{v}^{T}(t), \breve{w}^{T}(t)\right)^{T}$. Then subsystem (7b) can be rewritten in the matrix form as

$$
\dot{y}(t)=A y(t)+B y(t-\tau),
$$

where

$$
A=\left(\begin{array}{ccc}
O_{N-1} & I_{N-1} & O_{N-1} \\
-\alpha I_{N-1} & O_{N-1} & O_{N-1} \\
-(\alpha+1) I_{N-1} & O_{N-1} & -I_{N-1}
\end{array}\right) \text { and } B=\left(\begin{array}{ccc}
O_{N-1} & O_{N-1} & O_{N-1} \\
-\left(k_{1}+k_{2}\right) \widetilde{L} & O_{N-1} & -k_{2} \widetilde{L} \\
-\left(k_{1}+k_{2}\right) \widetilde{L} & O_{N-1} & -k_{2} \widetilde{L}
\end{array}\right)
$$

Remark 3 Since $\xi^{T}$ is the first row of $P^{-1}$, it follows from (5) that $\widetilde{x}_{1}(t)=\sum_{i=1}^{N} \xi_{i} x_{i}(t), \widetilde{v}_{1}(t)=$ $\sum_{i=1}^{N} \xi_{i} v_{i}(t)$ and $\widetilde{w}_{1}(t)=\sum_{i=1}^{N} \xi_{i} w_{i}(t)$. 
Lemma 2 The observer-based protocol (2) solves the second-order consensus problem of multi-agent system (1) if and only if system (8) is asymptotically stable. Moreover, as $t \rightarrow \infty$, the final states of all the agents in the network with dynamics (1) and (2) converge to some trajectory $\left(x^{*}(t), v^{*}(t), w^{*}(t)\right)^{T} \in \mathbb{R}^{3}$, that is, $x_{i}(t) \rightarrow x^{*}(t), v_{i}(t) \rightarrow v^{*}(t)$ and $w_{i}(t) \rightarrow w^{*}(t)$ for all $i=1, \ldots, N$, where $x^{*}(t), v^{*}(t)$ and $\left.w^{*}(t)\right)$ are given as follows:

1) If $\alpha>0, x^{*}(t)=\cos (\sqrt{\alpha} t) \xi^{T} x(0)+\frac{1}{\sqrt{\alpha}} \sin (\sqrt{\alpha} t) \xi^{T} v(0), v^{*}(t)=-\sqrt{\alpha} \sin (\sqrt{\alpha} t) \xi^{T} x(0)+\cos (\sqrt{\alpha} t) \xi^{T} v(0)$, and $w^{*}(t)=(-\cos (\sqrt{\alpha} t)-\sqrt{\alpha} \sin (\sqrt{\alpha} t)) \xi^{T} x(0)+\left(\cos (\sqrt{\alpha} t)-\frac{1}{\sqrt{\alpha}} \sin (\sqrt{\alpha} t)\right) \xi^{T} v(0)$.

2) If $\alpha=0, x^{*}(t)=\xi^{T} x(0)+t \xi^{T} v(0), v^{*}(t)=\xi^{T} v(0)$, and $w^{*}(t)=-\xi^{T} x(0)+(1-t) \xi^{T} v(0)$.

Proof. (Sufficiency) If system (8) is asymptotically stable, then one has $y(t)=\left(\breve{x}^{T}(t), \breve{v}^{T}(t), \breve{w}^{T}(t)\right)^{T} \rightarrow$ $0_{3(N-1)}$ as $t \rightarrow \infty$, that is, $\widetilde{x}_{i}(t) \rightarrow 0, \widetilde{v}_{i}(t) \rightarrow 0$ and $\widetilde{w}_{i}(t) \rightarrow 0$ for $i=2, \ldots, N$. It follows from $(5)$ that $x(t)=P \widetilde{x}(t), v(t)=P \widetilde{v}(t)$ and $w(t)=P \widetilde{w}(t)$. Since $1_{N}$ is the first column of matrix $P$, one can obtain $x_{i}(t)=\widetilde{x}_{1}(t), v_{i}(t)=\widetilde{v}_{1}(t)$ and $w_{i}(t)=\widetilde{w}_{1}(t)$ as $t \rightarrow \infty$, where $i=1, \ldots, N$, indicating that the second-order consensus in multi-agent system (1) with algorithm (2) is reached .

(Necessity) When multi-agent system (1) under observer-based protocol (2) achieves consensus, there should exist some $x^{*}(t) \in \mathbb{R}$ and $v^{*}(t) \in \mathbb{R}$ such that $x(t) \rightarrow 1_{N} x^{*}(t)$ and $v(t) \rightarrow 1_{N} v^{*}(t)$ as $t \rightarrow \infty$. By virtue of $P^{-1} P=I_{N}$, one can see that $q_{i}^{T} p_{1}=q_{i}^{T} 1_{N}=0, i=2, \ldots, N$. Then, in view of (5), it is easy to obtain $\widetilde{x}_{i}(t)=q_{i}^{T} x(t) \rightarrow q_{i}^{T} 1_{N} x^{*}(t) \rightarrow 0$ and $\widetilde{v}_{i}(t)=q_{i}^{T} v(t) \rightarrow q_{i}^{T} 1_{N} v^{*}(t) \rightarrow 0$ for $i=2, \ldots, N$, indicating $\breve{x}(t) \rightarrow 0_{N-1}$ and $\breve{v}(t) \rightarrow 0_{N-1}$ as $t \rightarrow \infty$. Recall that (8) is the compact matrix form of (7b). For a sufficiently large $t$, from $(7 \mathrm{~b})$ one has $k_{2} \widetilde{L} \breve{w}(t-\tau)=0$, yielding $\dot{\breve{w}}(t)=-\breve{w}(t)$. Then, one has $\breve{w}(t) \rightarrow 0_{N-1}$ as $t \rightarrow \infty$, that is, $\widetilde{w}_{i}(t) \rightarrow 0$ for $i=2, \ldots, N$. Hence, system (8) is asymptotically stable if multi-agent system (1) achieves consensus. Moreover, as $t \rightarrow \infty$, it follows from $w(t)=P \widetilde{w}(t)$ that $w_{i}(t) \rightarrow \widetilde{w}_{1}(t)$ for all $i=1, \ldots, N$, implying that the states of all the observers in (2) converge to some common trajectory.

Now, it is necessary to determine the final state $\left(x^{*}(t), v^{*}(t), w^{*}(t)\right)^{T}$ for the network with dynamics (1) and (2).

1) When $\alpha>0$, solving subsystem (7a) yields

$$
\begin{aligned}
\widetilde{x}_{1}(t)= & \cos (\sqrt{\alpha} t) \xi^{T} x(0)+\frac{1}{\sqrt{\alpha}} \sin (\sqrt{\alpha} t) \xi^{T} v(0), \widetilde{v}_{1}(t)=-\sqrt{\alpha} \sin (\sqrt{\alpha} t) \xi^{T} x(0)+\cos (\sqrt{\alpha} t) \xi^{T} v(0), \\
\widetilde{w}_{1}(t)= & \left(e^{-t}-\cos (\sqrt{\alpha} t)-\sqrt{\alpha} \sin (\sqrt{\alpha} t)\right) \xi^{T} x(0)+\left(-e^{-t}+\cos (\sqrt{\alpha} t)-\frac{1}{\sqrt{\alpha}} \sin (\sqrt{\alpha} t)\right) \xi^{T} v(0) \\
& +e^{-t} \xi^{T} w(0) .
\end{aligned}
$$

It follows from Remark 3 that $\widetilde{x}_{1}(t)=\xi^{T} x(t), \widetilde{v}_{1}(t)=\xi^{T} v(t)$ and $\widetilde{w}_{1}(t)=\xi^{T} w(t)$. Note that $e^{-t}$ converges to zero as $t \rightarrow \infty$. Considering $\xi^{T} 1_{N}=1$, one has $x^{*}(t)=\widetilde{x}_{1}(\infty), v^{*}(t)=\widetilde{v}_{1}(\infty)$ and $w^{*}(t)=\widetilde{w}_{1}(\infty)$, where $\widetilde{x}_{1}(\infty), \widetilde{v}_{1}(\infty)$ and $\widetilde{w}_{1}(\infty)$ can be easily obtained from $(9)$.

2 ) When $\alpha=0$, it follows from $(7 \mathrm{a})$ that $\widetilde{x}_{1}(t)=\widetilde{x}_{1}(0)+t \widetilde{v}_{1}(0)=\xi^{T} x(0)+t \xi^{T} v(0), \widetilde{v}_{1}(t)=\widetilde{v}_{1}(0)=$ $\xi^{T} v(0)$ and $\widetilde{w}_{1}(t)=\left(e^{-t}-1\right) \xi^{T} x(0)+\left(-e^{-t}+1-t\right) \xi^{T} v(0)+e^{-t} \xi^{T} w(0)$ and the final consensus state satisfies $x^{*}(t)=\widetilde{x}_{1}(\infty), v^{*}(t)=\widetilde{v}_{1}(\infty)$ and $w^{*}(t)=\widetilde{w}_{1}(\infty)$ when $t$ is sufficiently large.

By now, the proof is completed. 
Remark 4 From Lemma 2, one sees that the final consensus state $\left(x^{*}(t), v^{*}(t)\right)^{T}$ of multi-agent system (1) under algorithm (2) is determined by the topology, initial state and parameter $\alpha$ of network (1), and thus is independent of the time delay $\tau$. However, it will be shown that the time delay $\tau$ has a critical effect on the stability of multi-agent system (1) under observer-based consensus protocol (2).

According to the theory for linear time-delay systems, system (8) is asymptotically stable if and only if the roots of its characteristic equation are all located in the open left-half plane, that is, all the characteristic roots have negative real parts $[30,31,32]$. Let $\mu_{i}(1 \leq i \leq N)$ be the eigenvalues of $L$ satisfying $0=\left|\mu_{1}\right| \leq\left|\mu_{2}\right|<\ldots \leq\left|\mu_{N}\right|$. It follows from the similarity transformation $P^{-1} L P=\operatorname{diag}(0, \widetilde{L})$ that $\left\{\mu_{i} \mid i=2, \ldots, N\right\}$ is the eigenvalue set of matrix $\widetilde{L}$.

Let $\operatorname{CE}(\lambda, \tau)$ be the characteristic equation of system (8). Then $\operatorname{CE}(\lambda, \tau)$ can be calculated as follows

$$
\begin{aligned}
\operatorname{CE}(\lambda, \tau) & =\operatorname{det}\left(\lambda I_{3(N-1)}-A-B e^{-\lambda \tau}\right) \\
& =\operatorname{det}\left(\begin{array}{ccc}
\lambda I_{N-1} & -I_{N-1} & O_{N-1} \\
\alpha I_{N-1}+\left(k_{1}+k_{2}\right) \widetilde{L} e^{-\lambda \tau} & \lambda I_{N-1} & k_{2} \widetilde{L} e^{-\lambda \tau} \\
(\alpha+1) I_{N-1}+\left(k_{1}+k_{2}\right) \widetilde{L} e^{-\lambda \tau} & O_{N-1} & (\lambda+1) I_{N-1}+k_{2} \widetilde{L} e^{-\lambda \tau}
\end{array}\right) \\
& =\operatorname{det}\left(\begin{array}{ccc}
\lambda I_{N-1} & -I_{N-1} & O_{N-1} \\
\left(\lambda^{2}+\alpha\right) I_{N-1}+\left(k_{1}+k_{2}\right) \widetilde{L} e^{-\lambda \tau} & O_{N-1} & k_{2} \widetilde{L} e^{-\lambda \tau} \\
(\alpha+1) I_{N-1}+\left(k_{1}+k_{2}\right) \widetilde{L} e^{-\lambda \tau} & O_{N-1} & (\lambda+1) I_{N-1}+k_{2} \widetilde{L} e^{-\lambda \tau}
\end{array}\right) \\
& =\operatorname{det}\left(\begin{array}{cc}
\left(\lambda^{2}+\alpha\right) I_{N-1}+\left(k_{1}+k_{2}\right) \widetilde{L} e^{-\lambda \tau} & k_{2} \widetilde{L} e^{-\lambda \tau} \\
(\alpha+1) I_{N-1}+\left(k_{1}+k_{2}\right) \widetilde{L} e^{-\lambda \tau} & (\lambda+1) I_{N-1}+k_{2} \widetilde{L} e^{-\lambda \tau}
\end{array}\right) \\
& =\operatorname{det}\left(\begin{array}{cc}
\left(\lambda^{2}-1\right) I_{N-1} & -(\lambda+1) I_{N-1} \\
(\alpha+1) I_{N-1}+\left(k_{1}+k_{2}\right) \widetilde{L} e^{-\lambda \tau} & (\lambda+1) I_{N-1}+k_{2} \widetilde{L} e^{-\lambda \tau}
\end{array}\right) \\
& =(\lambda+1)^{N-1} \operatorname{det}\left(\lambda^{2} I_{N-1}+k_{2} \widetilde{L} \lambda e^{-\lambda \tau}+k_{1} \widetilde{L} e^{-\lambda \tau}+\alpha I_{N-1}\right) \\
& =(\lambda+1)^{N-1} \prod_{i=2}^{N}\left(\lambda^{2}+k_{2} \mu_{i} \lambda e^{-\lambda \tau}+k_{1} \mu_{i} e^{-\lambda \tau}+\alpha\right)=0,
\end{aligned}
$$

where $\mu_{i}$ is the $i$ th eigenvalue of $L$ with $2 \leq i \leq N$.

Let

$$
f_{i}(\lambda, \tau)=\lambda^{2}+\left(k_{1}+k_{2} \lambda\right) \mu_{i} e^{-\lambda \tau}+\alpha=0, i=2, \ldots, N .
$$

Then, $\operatorname{CE}(\lambda, \tau)=(\lambda+1)^{N-1} \prod_{i=2}^{N} f_{i}(\lambda, \tau)=0$.

Remark 5 It is interesting to note that $\lambda=-1$ is a root of the characteristic equation $\operatorname{CE}(\lambda, \tau)$ with multiplicity $N-1$. Therefore, the stability problem of third-order system (8) can be simply reduced to the stability analysis for a set of quasi-polynomials $f_{i}(\lambda, \tau)=0, i=2, \ldots, N$. Actually, the consensus protocol (2) is designed based on the separation principle for reduced-order observers to facilitate the study of the observer-based consensus of multi-agent system (1). 


\section{Observer-based consensus of multi-agent system without time delay}

This section considers the consensus in multi-agent system (1) under observer-based protocol (2) without time delay, and derives some necessary and sufficient conditions for reaching consensus.

When $\tau=0$, the characteristic equation $\operatorname{CE}(\lambda, 0)$ of system (8) has exactly $3(N-1)$ roots. Considering that $\lambda=-1$ is the root of multiplicity $N-1$ of $\operatorname{CE}(\lambda, \tau)$, it suffices to study the roots of $f_{i}(\lambda, 0)=0, i=$ $2, \ldots, N$ defined in (11) such that the stability of system (8) without time delay can be conveniently analyzed.

Lemma 3 [40] Given a complex-coefficient polynomial $p(\lambda)=\lambda^{2}+(a+\mathbf{i} b) \lambda+c+\mathbf{i} d$, where $a, b, c, d \in \mathbb{R}$, $p(\lambda)$ is asymptotically stable if and only if $a>0$ and $a b d+a^{2} c-d^{2}>0$.

The following result provides a necessary and sufficient condition to ensure the stability of system (8) without time delay.

Lemma 4 Assume that the digraph $\mathcal{G}$ of multi-agent system (1) contains a directed spanning tree. When $\tau=0$, system (8) is asymptotically stable if and only if the coupling strengths satisfy

$$
k_{1}>\max _{2 \leq i \leq N} \frac{-\alpha \operatorname{Re}\left(\mu_{i}\right)}{\left|\mu_{i}\right|^{2}}, k_{2}>\max _{2 \leq i \leq N} \frac{\left|k_{1} \operatorname{Im}\left(\mu_{i}\right)\right|}{\sqrt{\left(k_{1}\left|\mu_{i}\right|^{2}+\alpha \operatorname{Re}\left(\mu_{i}\right)\right) \operatorname{Re}\left(\mu_{i}\right)}} .
$$

Proof. When $\tau=0, f_{i}(\lambda, 0)=\lambda^{2}+k_{2} \mu_{i} \lambda+\left(k_{1} \mu_{i}+\alpha\right)=0, \forall i \in\{2, \ldots, N\}$. By Lemma 3 , the two roots of $f_{i}(\lambda, 0)=0$ have negative real parts if and only if the following two conditions hold simultaneously

$$
\begin{aligned}
& k_{2} \operatorname{Re}\left(\mu_{i}\right)>0, \\
& k_{2}^{2} \operatorname{Re}\left(\mu_{i}\right)\left[k_{1}\left(\operatorname{Re}^{2}\left(\mu_{i}\right)+\operatorname{Im}^{2}\left(\mu_{i}\right)\right)+\alpha \operatorname{Re}\left(\mu_{i}\right)\right]-k_{1}^{2} \operatorname{Im}^{2}\left(\mu_{i}\right)>0, i=2, \ldots, N .
\end{aligned}
$$

Since $\mathcal{G}$ has a directed spanning tree, by Lemma 1, all the nonzero eigenvalues of $L$ have positive real parts, that is, $\operatorname{Re}\left(\mu_{i}\right)>0$ for all $i=2, \ldots, N$. From (13a), one has $k_{2}>0$. Meanwhile, (13b) is equivalent to $k_{2}^{2} \operatorname{Re}\left(\mu_{i}\right)\left(k_{1}\left|\mu_{i}\right|^{2}+\alpha \operatorname{Re}\left(\mu_{i}\right)\right)>k_{1}^{2} \operatorname{Im}^{2}\left(\mu_{i}\right)$, indicating $k_{1}\left|\mu_{i}\right|^{2}+\alpha \operatorname{Re}\left(\mu_{i}\right)>0$ should hold for all $i=2, \ldots, N$, that is, $k_{1}>\max _{2 \leq i \leq N} \frac{-\alpha \operatorname{Re}\left(\mu_{i}\right)}{\left|\mu_{i}\right|^{2}}$. Then it follows from $k_{2}>0$ and (13b) that $k_{2}>$ $\max _{2 \leq i \leq N} \frac{\left|k_{1} \operatorname{Im}\left(\mu_{i}\right)\right|}{\sqrt{\left(k_{1}\left|\mu_{i}\right|^{2}+\alpha \operatorname{Re}\left(\mu_{i}\right)\right) \operatorname{Re}\left(\mu_{i}\right)}}$. Hence, all the roots of $\operatorname{CE}(\lambda, 0)$ has negative real parts if and only if condition (12) holds such that system (8) is asymptotically stable.

Remark 6 In fact, the existence of a directed spanning tree in the interaction digraph of multi-agent system (1) is a necessary condition to ensure the stability of system (8). If $\mathcal{G}$ does not have a directed spanning tree, the Laplacian matrix $L$ has at least two zero eigenvalues by Lemma 1 . Then there should exist an integer $p(2 \leq p \leq N)$ such that $\mu_{p}=0$, which indicates that conditions (13a) and (13b) in the proof of Lemma 4 will never be satisfied and thus system (8) is not asymptotically stable.

Remark 7 Based on the model parameter $\alpha$ and the nonzero eigenvalues of $L$ of multi-agent system (1), one can first choose an appropriate parameter $k_{1}$ according to condition (12). Then, with the selected $k_{1}$, the parameter $k_{2}$ can be easily designed such that $k_{2}>\max _{2 \leq i \leq N} \frac{\left|k_{1} \operatorname{Im}\left(\mu_{i}\right)\right|}{\sqrt{\left(k_{1}\left|\mu_{i}\right|^{2}+\alpha \operatorname{Re}\left(\mu_{i}\right)\right) \operatorname{Re}\left(\mu_{i}\right)}}$ holds. When $\alpha>0$, note that $k_{1}$ can be a negative number, but $k_{2}$ should be positive. 
Theorem 1 Suppose that the digraph $\mathcal{G}$ of multi-agent system (1) contains a directed spanning tree. Then

1) When $\tau=0$ and $\alpha>0$, the observer-based protocol (2) solves the consensus problem for multi-agent system (1) composed of harmonic oscillators if and only if condition (12) is satisfied. In particular, if $k_{1}=0$, coupled harmonic oscillators (1) with algorithm (2) can always achieve consensus for any $k_{2}>0$.

2) When $\tau=0$ and $\alpha=0$, the consensus in multi-agent system (1) with double-integrator dynamics using observer-based protocol (2) can be reached if and only if $k_{1}>0$ and $k_{2}>\max _{2 \leq i \leq N} \frac{\left|k_{1} \operatorname{Im}\left(\mu_{i}\right)\right|}{\left|\mu_{i}\right| \sqrt{k_{1} \operatorname{Re}\left(\mu_{i}\right)}}$.

Proof. 1) When $\alpha>0$, each agent in network (1) is a harmonic oscillator. The first statement follows directly from Lemmas 2 and 4 . Obviously, one can choose $k_{1}=0$ and $k_{2}>0$ to satisfy condition (12) such that the consensus in the network can always be achieved.

2) When $\tau=0$ and $\alpha=0$, it follows from condition (12), Lemmas 2 and 4 that system (8) is asymptotically stable if and only if $k_{1}>0$ and $k_{2}>\max _{2 \leq i \leq N} \frac{\left|k_{1} \operatorname{Im}\left(\mu_{i}\right)\right|}{\left|\mu_{i}\right| \sqrt{k_{1} \operatorname{Re}\left(\mu_{i}\right)}}$ are satisfied such that the consensus in network (1) with double-integrator dynamics using algorithm (2) can be reached.

By now, the proof is complete

\section{Observer-based consensus of multi-agent system with time delay}

In the previous section, the consensus in delay-free multi-agent system (1) using algorithm (2) has been investigated. This section further studies the consensus in network (1) under observer-based protocol (2) with communication delay.

When $\tau=0$, the characteristic equation $\operatorname{CE}(\lambda, 0)$ of system (8) has exactly $3(N-1)$ roots. However, for any $\tau>0, \operatorname{CE}(\lambda, \tau)$ is a transcendental equation with an infinite number of roots [30,31,32]. Hence, it is literally impossible to compute all the roots of $\mathrm{CE}(\lambda, \tau)$. Fortunately, some techniques for linear time-delay systems can be adopted to address the consensus problem for multi-agent system (1) using algorithm (2) with time delay.

The following result, which follows directly from Corollary 2.4 in [32], is important to discuss the root distribution of the characteristic equation $\mathrm{CE}(\lambda, \tau)$.

Lemma 5 [32] As time delay $\tau$ varies, the number of the roots of $\operatorname{CE}(\lambda, \tau)$ with nonnegative real parts can change only if a root appears on or crosses the imaginary axis.

Remark 8 Let $\mathrm{NU}(\tau)$ denote the number of the roots of $\mathrm{CE}(\lambda, \tau)$ with nonnegative real parts. From Lemma 5, one knows that the consensus in multi-agent system (1) under observer-based protocol (2) can be achieved if and only if $\mathrm{NU}(\tau)=0$ in some time-delay intervals. Note that $\mathrm{NU}(\tau)$ may change when $\mathrm{CE}(\lambda, \tau)$ has some purely imaginary roots. Hence, to study the stability of system (8) with time delay, it is necessary to discuss the conditions such that $\mathrm{CE}(\lambda, \tau)$ has purely imaginary roots.

The following lemma provides some conditions, under which the characteristic equation $\operatorname{CE}(\lambda, \tau)$ of system (8) has a set of purely imaginary roots. 
Lemma 6 Suppose that $\mathcal{G}$ contains a directed spanning tree. Let the coupling strengths satisfy

$$
k_{1}>\frac{\alpha}{\min _{2 \leq i \leq N}\left|\mu_{i}\right|}, k_{2}>0 .
$$

Then, $\operatorname{CE}(\lambda, \tau)$ has a set of purely imaginary roots $\left\{ \pm \mathbf{i} \omega_{i} \mid i=2, \ldots, N\right\}$ if and only if

$$
\tau \in \Psi \triangleq\left\{\frac{2 k \pi+\theta_{i}}{\omega_{i}} \mid i \in S_{1}, k \in \mathbb{Z}^{+}\right\} \bigcup\left\{\frac{(2 k+2) \pi+\theta_{i}}{\omega_{i}} \mid i \in S_{2}, k \in \mathbb{Z}^{+}\right\},
$$

where

$$
\begin{aligned}
& \omega_{i}=\sqrt{\frac{2 \alpha+k_{2}^{2}\left|\mu_{i}\right|^{2}+\sqrt{k_{2}^{4}\left|\mu_{i}\right|^{4}+4 \alpha k_{2}^{2}\left|\mu_{i}\right|^{2}+4 k_{1}^{2}\left|\mu_{i}\right|^{2}}}{2}}, i \in\{2, \ldots, N\}, \\
& \theta_{i}=\operatorname{Arg}\left(\mu_{i}\right)+\arctan \frac{k_{2} \omega_{i}}{k_{1}} \text { with }-\pi / 2<\operatorname{Arg}\left(\mu_{i}\right)<\pi / 2, \quad i \in\{2, \ldots, N\}, \\
& S_{1}=\left\{i \mid \theta_{i} \geq 0, i=2, \ldots, N\right\} \text { and } S_{2}=\left\{i \mid \theta_{i}<0, i=2, \ldots, N\right\} .
\end{aligned}
$$

Proof. Let $\lambda=\mathbf{i} \omega_{i}\left(\omega_{i}>0\right)$ be the root of $f_{i}(\lambda, \tau)=0$ defined in (11). It follows from $f_{i}\left(\mathbf{i} \omega_{i}, \tau\right)=0$ that

$$
\omega_{i}^{2}-\alpha=\mu_{i}\left(k_{1}+\mathbf{i} \omega_{i} k_{2}\right) e^{-\mathbf{i} \omega_{i} \tau} .
$$

Let $\mu_{i}=\left|\mu_{i}\right| e^{\mathbf{i} \operatorname{Arg}\left(\mu_{i}\right)}$. Considering that $k_{1}, k_{2}$ and $\omega_{i}$ are all positive numbers, one can let $k_{1}+\mathbf{i} \omega_{i} k_{2}=$ $\sqrt{k_{1}^{2}+k_{2}^{2} \omega_{i}^{2}} e^{\mathrm{i} \arctan \frac{k_{2} \omega_{i}}{k 1}}$ with $0<\arctan \frac{k_{2} \omega_{i}}{k 1}<\pi / 2$. Then (19) can be rewritten as

$$
\omega_{i}^{2}-\alpha=\left|\mu_{i}\right| \sqrt{k_{1}^{2}+k_{2}^{2} \omega_{i}^{2}} e^{\mathbf{i}\left(\theta_{i}-\omega_{i} \tau\right)},
$$

where $\theta_{i}=\operatorname{Arg}\left(\mu_{i}\right)+\arctan \frac{k_{2} \omega_{i}}{k 1}$.

Taking modulus on both sides of (20) yields

$$
\left|\omega_{i}^{2}-\alpha\right|=\left|\mu_{i}\right| \sqrt{k_{1}^{2}+k_{2}^{2} \omega_{i}^{2}} .
$$

By condition (14), $k_{1}>\frac{\alpha}{\min _{2 \leq i \leq N}\left|\mu_{i}\right|}$ holds ensuring that $\omega_{i}$ can be uniquely determined by (16), indicating $\omega_{i}^{2}>\alpha$. Then it follows from (20) and (21) that

$$
e^{\mathbf{i}\left(\theta_{i}-\omega_{i} \tau\right)}=1 .
$$

Hence, $\omega_{i} \tau=2 k \pi+\theta_{i}, k \in \mathbb{Z}^{+}$. When $\mathcal{G}$ contains a directed spanning tree, by Lemma 1 , one has $\operatorname{Re}\left(\mu_{i}\right)>0, i=2, \ldots, N$, indicating $-\pi / 2<\operatorname{Arg}\left(\mu_{i}\right)<\pi / 2$. Hence, $\theta_{i}=\operatorname{Arg}\left(\mu_{i}\right)+\arctan \frac{k_{2} \omega_{i}}{k_{1}}$ may be negative. Meanwhile, note that $\tau \geq 0$ should hold. By partitioning network nodes into two set $S_{1}$ and $S_{2}$ defined in (18), one sees that $\mathbf{i} \omega_{i}$ is a purely imaginary root of $f_{i}(\lambda, \tau)=0$ only when $\tau=\frac{2 k \pi+\theta_{i}}{\omega_{i}}$ if $i \in S_{1}$ or $\tau=\frac{(2 k+2) \pi+\theta_{i}}{\omega_{i}}$ if $i \in S_{2}$, where $k \in \mathbb{Z}^{+}$.

Meanwhile, one can show that $-\mathbf{i} \omega_{i}$ is a purely imaginary root of $f_{j}(\lambda, \tau)=0$ where $j \in\{2, \ldots, N\}$ is an integer to be determined. From $f_{i}\left(\mathbf{i} \omega_{i}, \tau\right)=0$, one has $\overline{f_{i}\left(\mathbf{i} \omega_{i}, \tau\right)}=0$. Recall $\mu_{i}=\left|\mu_{i}\right| e^{\mathbf{i} A r g\left(\mu_{i}\right)}$. Taking conjugate on both sides of (19) leads to

$$
\omega_{i}^{2}-\alpha=\left|\mu_{i}\right|\left(k_{1}-\mathbf{i} \omega_{i} k_{2}\right) e^{\mathbf{i}\left(-\operatorname{Arg}\left(\mu_{i}\right)+\omega_{i} \tau\right)} .
$$


Consider the following two cases.

Case 1. $\operatorname{Im}\left(\mu_{i}\right)=0$ : Obviously, $\operatorname{Arg}\left(\mu_{i}\right)=0, i=2, \ldots, N$. It follows from $(22)$ that $-\omega_{i}^{2}+\mu_{i}\left(k_{1}-\right.$ $\left.\mathbf{i} \omega_{i} k_{2}\right) e^{\mathbf{i} \omega_{i} \tau}+\alpha=0$, which means that $f_{i}\left(-\mathbf{i} \omega_{i}, \tau\right)=0$.

Case 2. $\operatorname{Im}\left(\mu_{i}\right) \neq 0$ : It is well known that the complex eigenvalues of $L$ should occur in conjugate pairs located in the first and the fourth quadrants, respectively. Then, for any complex eigenvalue $\mu_{i}$, there should exist an integer $j(2 \leq j \leq N)$ such that

$$
\mu_{j}=\overline{\mu_{i}} \text { and } \operatorname{Arg}\left(\mu_{j}\right)=-\operatorname{Arg}\left(\mu_{i}\right) \text {, }
$$

It follows from (16) and (23) that

$$
\omega_{j}=\omega_{i}
$$

Substituting (23) and (24) into (22) yields

$$
\omega_{j}^{2}-\alpha=\left|\mu_{j}\right|\left(k_{1}-\mathbf{i} \omega_{j} k_{2}\right) e^{\mathbf{i}\left(\operatorname{Arg}\left(\mu_{j}\right)+\omega_{j} \tau\right)}=\mu_{j}\left(k_{1}-\mathbf{i} \omega_{j} k_{2}\right) e^{\mathbf{i} \omega_{j} \tau},
$$

which clearly indicates $f_{j}\left(-\mathbf{i} \omega_{j}, \tau\right)=0$. Recalling $\omega_{j}=\omega_{i}$, one has $f_{j}\left(-\mathbf{i} \omega_{i}, \tau\right)=0$.

Combining the discussions for the above two cases, one knows that $-\mathbf{i} \omega_{i}$ is a purely imaginary root of $f_{j}(\lambda, \tau)=0$. By now, one can conclude that $\operatorname{CE}(\lambda, \tau)$ has a set of purely imaginary roots $\left\{ \pm \mathbf{i} \omega_{i} \mid i=\right.$ $2, \ldots, N\}$ if and only if $\tau \in \Psi$.

With the increase of time delay, the purely imaginary roots of $\mathrm{CE}(\lambda, \tau)$ may cross the imaginary axis and may affect the stability of system (8). The following result addresses the moving directions of the purely imaginary roots of $\mathrm{CE}(\lambda, \tau)$.

Lemma 7 Let $\tau \in \Psi$ such that $\operatorname{CE}(\lambda, \tau)$ has purely imaginary roots, where $\Psi$ is defined in (15). With a small increase of time delay, all the purely imaginary roots of $\mathrm{CE}(\lambda, \tau)$ associated with $\tau$ will move to the open right-half plane.

Proof. By Lemma 6, one knows that $\operatorname{CE}(\lambda, \tau)$ has purely imaginary roots only when $\tau \in \Psi$. Hence, it is necessary to determine the moving directions of these roots as the time delay $\tau$ is increased. Let $\lambda$ be the solution of $f_{i}(\lambda, \tau)=0, i \in\{2, \ldots, N\}$. It follows from $f_{i}(\lambda, \tau)=0$ that

$$
\lambda^{2}+\left(k_{1}+k_{2} \lambda\right) \mu_{i} e^{-\lambda \tau}+\alpha=0 .
$$

Taking the derivative with respect to $\tau$ in (26) yields

$$
\frac{d \lambda}{d \tau}=\frac{\lambda\left(k_{1}+k_{2} \lambda\right) \mu_{i} e^{-\lambda \tau}}{2 \lambda+\mu_{i}\left[k_{2}-\tau\left(k_{1}+k_{2} \lambda\right)\right] e^{-\lambda \tau}} .
$$

From (26), one has

$$
\left(k_{1}+k_{2} \lambda\right) \mu_{i} e^{-\lambda \tau}=-\lambda^{2}-\alpha .
$$

Then (27) can be rewritten as

$$
\frac{d \lambda}{d \tau}=\frac{-\lambda\left(\lambda^{2}+\alpha\right)}{2 \lambda+\tau\left(\lambda^{2}+\alpha\right)+k_{2} \mu_{i} e^{-\lambda \tau}} .
$$


Let $q(\lambda)=2 \lambda+\tau\left(\lambda^{2}+\alpha\right)+k_{2} \mu_{i} e^{-\lambda \tau}$. It follows from (28) and (29) that

$$
\begin{aligned}
\frac{d \lambda}{d \tau} & =\frac{-\lambda\left(\lambda^{2}+\alpha\right) \overline{q(\lambda)}}{|q(\lambda)|^{2}} \\
& =\frac{-2|\lambda|^{2}\left(\lambda^{2}+\alpha\right)-\tau \lambda\left|\lambda^{2}+\alpha\right|^{2}-k_{2} \lambda\left(\lambda^{2}+\alpha\right) \overline{\mu_{i} e^{-\lambda \tau}}}{|q(\lambda)|^{2}} \\
& =\frac{-2|\lambda|^{2}\left(\lambda^{2}+\alpha\right)-\tau \lambda\left|\lambda^{2}+\alpha\right|^{2}+k_{2} \lambda\left(k_{1}+k_{2} \lambda\right)\left|\mu_{i} e^{-\lambda \tau}\right|^{2}}{|q(\lambda)|^{2}} .
\end{aligned}
$$

When $\tau \in \Psi$, by the proof of Lemma 6 , one knows that $\lambda=\mathbf{i} \omega_{i}$ is the purely imaginary root of $f_{i}(\lambda, \tau)=0$, where $i \in\{2, \ldots, N\}$. It follows from (16) and (30) that

$$
\begin{aligned}
\operatorname{sign}\left(\operatorname{Re}\left(\left.\frac{d \lambda}{d \tau}\right|_{\tau \in \Psi, \lambda=\mathrm{i} \omega_{i}}\right)\right) & =\operatorname{sign}\left(-2 \omega_{i}^{2}\left(-\omega_{i}^{2}+\alpha\right)-k_{2}^{2} \omega_{i}^{2}\left|\mu_{i}\right|^{2}\right) \\
& =\operatorname{sign}\left(\omega_{i}^{2} \sqrt{k_{2}^{4}\left|\mu_{i}\right|^{4}+4 \alpha k_{2}^{2}\left|\mu_{i}\right|^{2}+4 k_{1}^{2}\left|\mu_{i}\right|^{2}}\right)=1>0 .
\end{aligned}
$$

Meanwhile, $\lambda=-\mathbf{i} \omega_{i}$ is the purely imaginary root of $f_{j}(\lambda, \tau)=0$ where $j$ is some integer in $\{2, \ldots, N\}$. Following the similar line for the case $\lambda=\mathbf{i} \omega_{i}$, it is easy to show that $\operatorname{sign}\left(\operatorname{Re}\left(\left.\frac{d \lambda}{d \tau}\right|_{\tau \in \Psi, \lambda=-\mathbf{i} \omega_{i}}\right)\right)>0$ holds. Hence, all the purely imaginary roots of $\operatorname{CE}(\lambda, \tau)$ associated with $\tau$ will cross the imaginary axis and enter the open right-half plane with a small increase of the time delay. This completes the proof.

By Lemma 6 , let $\tau^{*}$ be the minimum time-delay belonging to the set $\Psi$ such that $\operatorname{CE}(\lambda, \tau)$ has purely imaginary roots. It follows from (15) that

$$
\tau^{*}=\min \Psi=\min \left\{\min _{i \in S_{1}} \frac{\theta_{i}}{\omega_{i}}, \min _{i \in S_{2}} \frac{2 \pi+\theta_{i}}{\omega_{i}}\right\} .
$$

Remark 9 When $\tau=\tau^{*}, \operatorname{CE}(\lambda, \tau)$ has purely imaginary roots and system (8) is not asymptotically stable, and then the consensus in multi-agent system (1) with algorithm (2) can not be reached.

Theorem 2 Suppose that the topology of multi-agent system (1) contains a directed spanning tree.

1) Let

$$
k_{1}>\frac{\alpha}{\min _{2 \leq i \leq N}\left|\mu_{i}\right|} \text { and } k_{2}>\max _{2 \leq i \leq N} \frac{\left|k_{1} \operatorname{Im}\left(\mu_{i}\right)\right|}{\sqrt{\left(k_{1}\left|\mu_{i}\right|^{2}+\alpha \operatorname{Re}\left(\mu_{i}\right)\right) \operatorname{Re}\left(\mu_{i}\right)}} .
$$

When $\alpha>0$, the consensus in multi-agent system (1) composed of harmonic oscillators under observerbased protocol (2) can be reached if and only if $\tau \in\left[0, \tau^{*}\right)$, where $\tau^{*}$ is defined in (32).

2) Let $\alpha=0, k_{1}>0$ and $k_{2}>\max _{2 \leq i \leq N} \frac{\left|k_{1} \operatorname{Im}\left(\mu_{i}\right)\right|}{\left|\mu_{i}\right| \sqrt{k_{1} \operatorname{Re}\left(\mu_{i}\right)}}$. Using algorithm (2), multi-agent system (1) with double-integrator dynamics can achieve consensus if and only if $\tau \in\left[0, \tau^{*}\right)$ with $\tau^{*}$ being defined by (32).

Proof. 1) When $\alpha>0$ and $\tau=0$, condition (33) guarantees that condition (12) in Theorem 1 is satisfied such that the consensus in delay-free coupled harmonic oscillators (1) using algorithm (2) is reached. Let $\tau$ vary from 0 to $\tau^{*}$. According to Lemma $6, \operatorname{CE}(\lambda, \tau)$ has no purely imaginary root until $\tau=\tau^{*}$ under conditions (14) and (33). Moreover, for any $\tau>\tau^{*}, \operatorname{CE}(\lambda, \tau)$ should have at least two roots with positive real parts in view of Lemma 7 and its proof. Therefore, system (8) is asymptotically stable if and only if $\tau \in\left[0, \tau^{*}\right)$ such that the consensus in multi-agent system (1) is achieved. 
2) When $\tau=0$ and $\alpha=0$, by Theorem 1, one knows that coupled double-integrators (1) under delayfree protocol (2) can achieve consensus if $k_{1}>0$ and $k_{2}>\max _{2 \leq i \leq N} \frac{\left|k_{1} \operatorname{Im}\left(\mu_{i}\right)\right|}{\left|\mu_{i}\right| \sqrt{k_{1} \operatorname{Re}\left(\mu_{i}\right)}}$. Following the similar line in part 1 , it is easy to show that the consensus in coupled double integrators (1) under protocol (2) can be reached if and only if $\tau \in\left[0, \tau^{*}\right)$.

\section{$6 \quad$ Numerical results}

In this section, some numerical results are provided to illustrate the effectiveness of the theoretical analysis. Under observer-based protocol (2), consider multi-agent system (1) composed of five agents, whose Laplacian matrix is given by $L=\left(\begin{array}{ccccc}1 & 0 & 0 & 0 & -1 \\ -1 & 2 & 0 & 0 & -1 \\ 0 & -1 & 1 & 0 & 0 \\ 0 & 0 & -1 & 1 & 0 \\ -1 & 0 & 0 & -1 & 2\end{array}\right)$. The eigenvalues of matrix $L$ are obtained as $0,1.1226+0.7449 \mathbf{i}, 1.1226-0.7449 \mathbf{i}, 2$ and 2.7549 . By Lemma 1 , one knows that the network topology contains a directed spanning tree.

\subsection{Consensus in a network of harmonic oscillators}

For multi-agent system (1), let $\alpha=1.0$, indicating that each agent is a harmonic oscillator.

Case 1: $\tau=0$. When time delay is equal to zero, by Theorem 1, the consensus in coupled harmonic oscillators (1) using algorithm (2) can be achieved if and only if condition (12) is satisfied. To design the coupling strengths $k_{1}$ and $k_{2}$, let $k_{1}=-0.25$ such that $k_{1}>\max _{2 \leq i \leq N} \frac{-\alpha \operatorname{Re}\left(\mu_{i}\right)}{\left|\mu_{i}\right|^{2}}=-0.3630$ holds. By condition (12), the parameter $k_{2}$ should be greater than $\max _{2 \leq i \leq N} \frac{\left|k_{1} \operatorname{Im}\left(\mu_{i}\right)\right|}{\sqrt{\left(k_{1}\left|\mu_{i}\right|^{2}+\alpha \operatorname{Re}\left(\mu_{i}\right)\right) \operatorname{Re}\left(\mu_{i}\right)}}=0.2149$. Fig. 1(a) indicates that the consensus can not be achieved when $k_{2}=0.2<0.2149$. One can see that the consensus is successfully reached when $k_{2}=0.28>0.2149$ from Fig. 1(b).

Case 2: $\tau>0$. Now consider the consensus in coupled harmonic oscillators with communication delay. Some simple calculations give $\frac{\alpha}{\min _{2<i<N}\left|\mu_{i}\right|}=0.7423$. Let $k_{1}=0.75$ and $k_{2}=1$ to satisfy condition
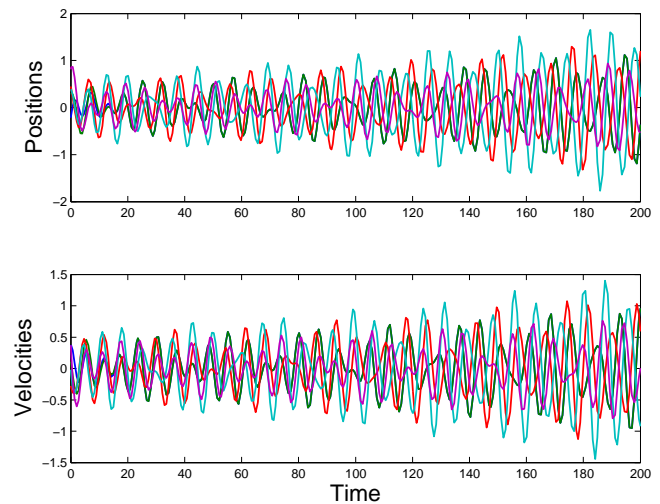

(a) $k_{1}=-0.25$ and $k_{2}=0.2$
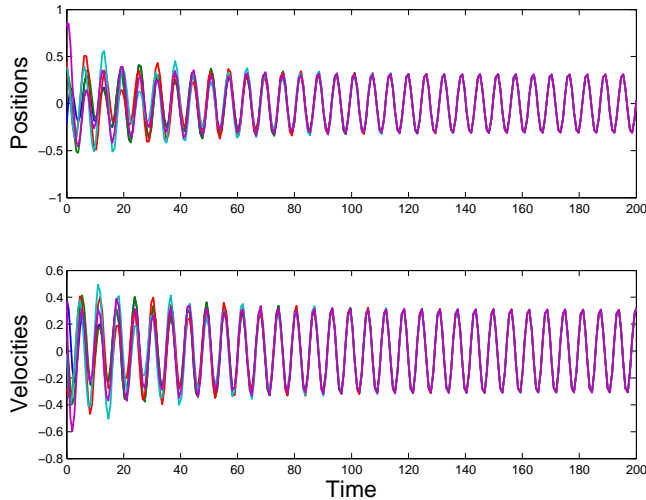

(b) $k_{1}=-0.25$ and $k_{2}=0.28$

Figure 1: Position and velocity states of coupled harmonic oscillators without time delay 
(33). It follows from (32) that $\tau^{*}=0.3165$. By Theorem 2 , the consensus can be achieved if and only if the time delay is smaller than $\tau^{*}$. Fig. 2(a) and Fig. 2(b) show that the consensus is achieved when $\tau=0.31$, but can not be reached when $\tau=0.32$.

\subsection{Consensus in a network of double integrators}

Let $\alpha=0$ such that multi-agent system (1) has double-integrator dynamics.

Case 1: $\tau=0$. By Theorem 1, the consensus problem in delay-free coupled double integrators can be solved if and only if $k_{1}>0$ and $k_{2}>\max _{2 \leq i \leq N} \frac{\left|k_{1} \operatorname{Im}\left(\mu_{i}\right)\right|}{\left|\mu_{i}\right| \sqrt{k_{1} \operatorname{Re}\left(\mu_{i}\right)}}$. Letting $k_{1}=0.5$, one knows that $k_{2}>0.3690$ should hold. Fig. 3 (a) shows that the consensus can not be achieved when $k_{2}=0.36$. Fig. 3(b) confirms that the consensus is reached when $k_{2}=0.45$.

Case 2: $\tau>0$. Now consider the consensus in coupled double integrators with communication delay. Let $k_{1}=0.5$ and $k_{2}=0.8$ to satisfy condition in Theorem 1 such that consensus can be achieved when $\tau=0$. It follows from (32) that $\tau^{*}=0.4198$. The state evolutions of the network for $\tau=0.41$ and $\tau=0.422$ are shown in Fig. 4(a) and Fig. 4(b), respectively, from which one sees that according to Theorem 2, the consensus in coupled double integrators can be achieved if and only if $\tau \in[0,0.4198)$.

\section{Conclusions}

Without measuring velocity states of the agents, this paper proposes a unified observer-based consensus protocol for second-order multi-agent systems composed of harmonic oscillators or double integrators. By using matrix transformation and system decomposition, the observer-based consensus problem is converted to the stability problem for a set of second-order quasi-polynomials. The coupling strengths of multi-agent system are conveniently designed based on the model parameter $\alpha$ and the nonzero eigenvalues of the Laplacian matrix. Some necessary and sufficient conditions are derived for reaching second-order consensus in multi-agent systems without time delay. By analyzing the motion directions of purely imaginary roots of the characteristic equation of the partitioned subsystem, we have further shown that second-order consensus can be achieved if and only if the communication delay is less than a positive
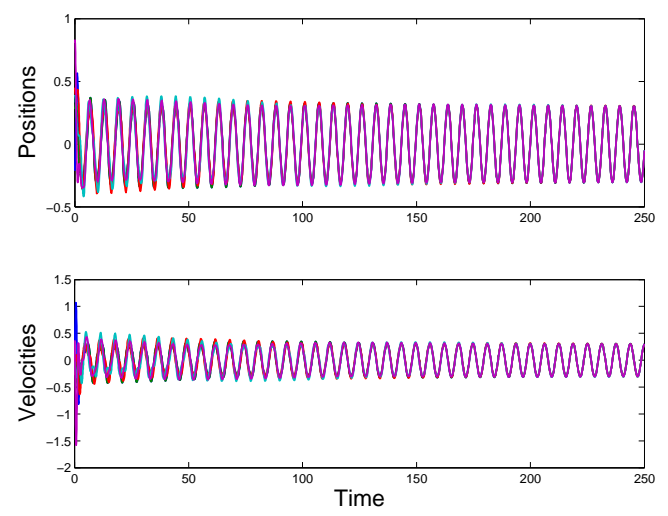

(a) $\tau=0.31$
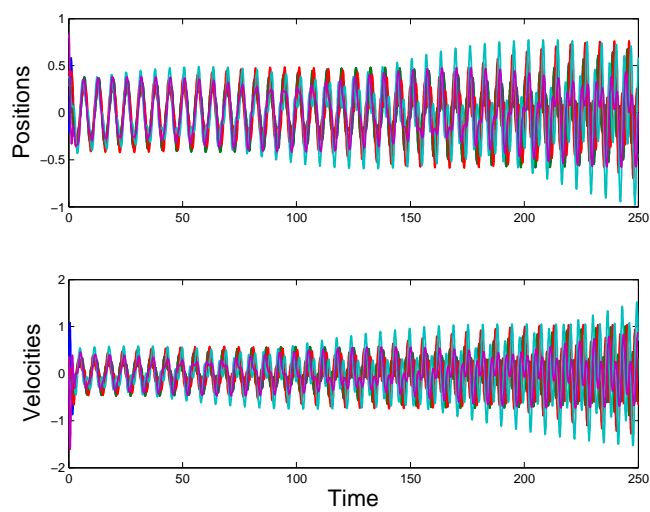

(b) $\tau=0.32$

Figure 2: Position and velocity states of coupled harmonic oscillators with time delay 

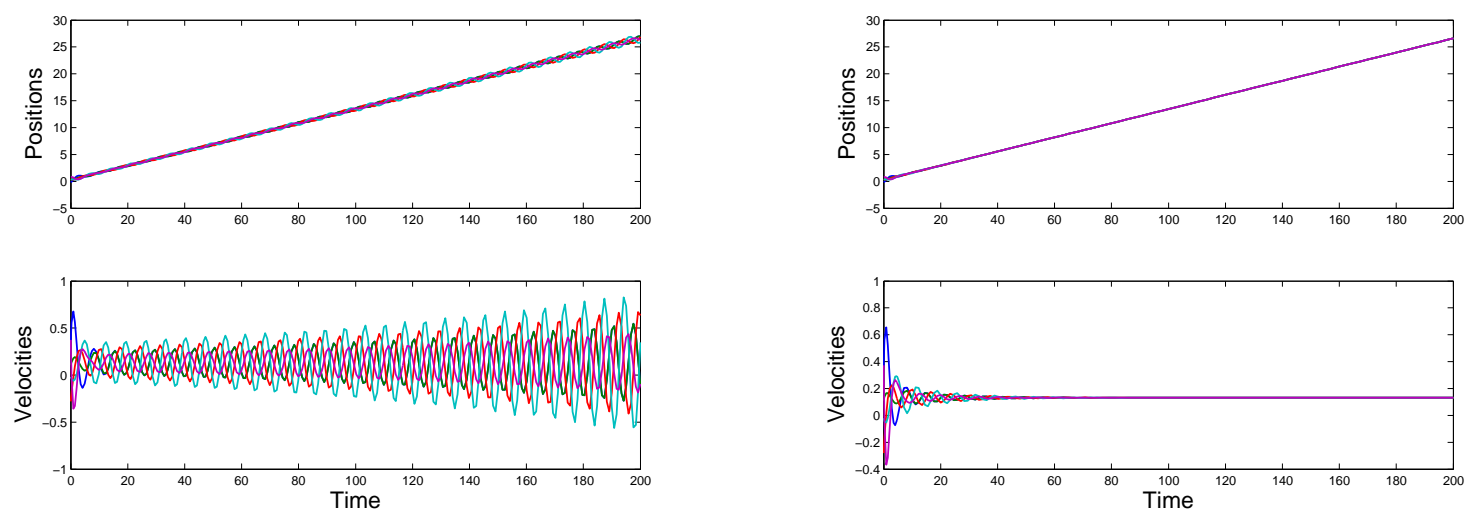

(a) $k_{1}=0.5$ and $k_{2}=0.36$

(b) $k_{1}=0.5$ and $k_{2}=0.45$

Figure 3: Position and velocity states of coupled double integrators without time delay
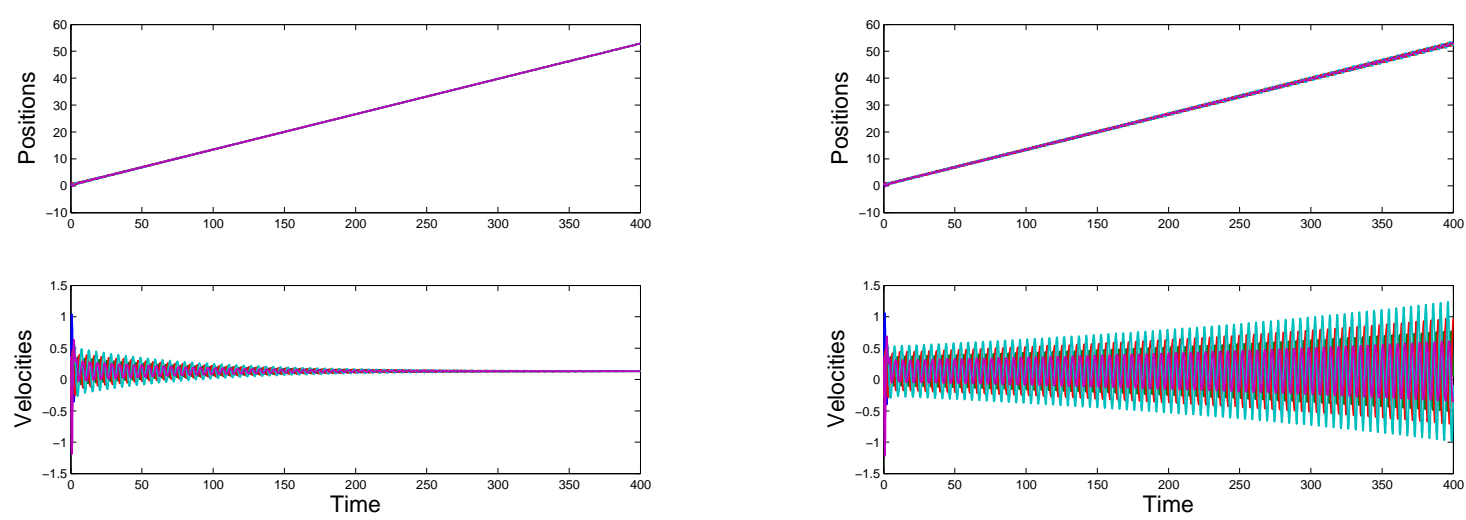

(a) $\tau=0.41$

(b) $\tau=0.422$

Figure 4: Position and velocity states of coupled double integrators with time delay 
threshold. It should be pointed out that when the consensus in multi-agent system is reached, the final consensus state is only determined by the model parameter $\alpha$, network topology and initial state of multiagent system. In out future work, we would like to extend the theoretical results of this paper to secondorder multi-agent systems with multiple communication delays and dynamically changing topologies.

\section{Acknowledgments}

This work was jointly supported by the National Science Foundation of China under Grants 61273218, 61304172, 61304258, 61333011 and 61273075, Scientific Research Fund of Henan Provincial Education Department under Grant 15A413004, the Natural Science Foundation of Henan Province of China under Grants 122102210027 and 15211, and the Research Foundation for Advanced Talents of Henan University of Technology under Grant 31401548.

\section{References}

[1] R. Olfati-Saber, R. M. Murray, Consensus problems in networks of agents with switching topology and time-delays, IEEE Transactions on Automatic Control 49(9)(2004)1520-1533.

[2] W. Ren, R. W. Beard, Consensus seeking in multiagent systems under dynamically changing interaction topologies, IEEE Transactions on Automatic Control 50(5)(2005)655-661.

[3] J. Lu, D. W. C. Ho, J. Kurths, Consensus over directed static networks with arbitrary finite communication delays, Physical Review E 80(6)(2009)066121.

[4] W. Ren, E. Atkins, Distributed multi-vehicle coordinated control via local information exchange, International Journal of Robust and Nonlinear Control 17(10-11)(2007)1002-1033.

[5] W. Yu, G. Chen, M. Cao, Some necessary and sufficient conditions for second-order consensus in multi-agent dynamical systems, Automatica 46(6)(2010)1089-1095.

[6] Z. Meng, W. Ren, Y. Cao, Z. You, Leaderless and leader-following consensus with communication and input delays under a directed network topology, IEEE Transactions on Systems, Man, and Cybernetics-Part B: Cybernetics 41(1)(2011)75-88.

[7] G. Wen, Z. Duan, W. Yu, G. Chen, Consensus in multi-agent systems with communication constraints, International Journal of Robust and Nonlinear Control 22(2)(2012)170-182.

[8] Q. Song, F. Liu, J. Cao, W. Yu, M-Matrix Strategies for pinning-controlled leader-following consensus in multiagent systems with nonlinear dynamics, IEEE Transactions on Cybernetics 43(6)(2013)1688-1697.

[9] G. Wen, Z. Duan, W. Yu, G. Chen, Consensus of second-order multi-agent systems with delayed nonlinear dynamics and intermittent communications, International Journal of Control 86(2) (2013)322331. 
[10] J. Xi, N. Cai, Y. Zhong, Consensus problems for high-order linear time-invariant swarm systems, Physica A 389(24)(2010)5619-5627.

[11] Z. Li, X. Liu, P. Lin, W. Ren, Consensus of linear multi-agent systems with reduced-order observerbased protocols, Systems \& Control Letters 60(7)(2011)510-516.

[12] G. Wen, Z. Duan, G. Chen, W. Yu, Consensus tracking of multi-agent systems with Lipschitz-type node dynamics and switching topologies, IEEE Transactions on Circuits and Systems-I: Regular Papers 61(2)(2014)499-511.

[13] B. Zhou, C. Xu, G. Duan, Distributed and truncated reduced-order observer based output feedback consensus of multi-agent systems, IEEE Transactions on Automatic Control 59(8)(2014)2264-2270.

[14] Z. Yu, H. Jiang, C. Hu, Leader-following consensus of fractional-order multi-agent systems under fixed topology, Neurocomputing 149(2015)613-620.

[15] C. Song, J. Cao, Y. Liu, Robust consensus of fractional-order multi-agent systems with positive real uncertainty via second-order neighbors information, Neurocomputing 165(2015)293-299.

[16] R. Olfati-Saber, Flocking for multi-agent dynamic systems: Algorithms and theory, IEEE Transactions on Automatic Control 51(3)(2006)401-420.

[17] H. Su, X. Wang, Z. Lin, Flocking of multi-agents with a virtual leader, IEEE Transactions on Automatic Control 54(2)(2009)293-307.

[18] T. Balch, R. C. Arkin, Behavior-based formation control for multirobot teams, IEEE Transactions on Robotics and Automation 14(6)(1998)926-939.

[19] Z. Lin, B. Francis, M. Maggiore, Necessary and sufficient graphical conditions for formation control of unicycles, IEEE Transactions on Automatic Control 50(1)(2005)121-127.

[20] X. Yang, Z. Yang, Synchronization of TS fuzzy complex dynamical networks with time-varying impulsive delays and stochastic effects, Fuzzy Sets and Systems 235(2014) 25-43.

[21] X. Liu, L. Ping, T. Chen, Cluster synchronization for delayed complex networks via periodically intermittent pinning control, Neurocomputing 162 (2015) 191-200.

[22] X. Yang, Q. Song, J. Liang, B. He, Finite-time synchronization of coupled discontinuous neural networks with mixed delays and nonidentical perturbations, Journal of the Franklin Institute $352(10)(2015) 4382-4406$.

[23] C. Hu, J. Yu, H. Jiang, Finite-time synchronization of delayed neural networks with Cohen-Grossberg type based on delayed feedback control, Neurocomputing 143(2014)90-96.

[24] W. Li and M. W. Spong, Stability of general coupled inertial agents, IEEE Transactions on Automatic Control 55(6)(2010)1411-1416. 
[25] W. Ren, Synchronization of coupled harmonic oscillators with local interaction, Automatica 44(12)(2008)3195-3200.

[26] H. Su, X. Wang, Z. Lin, Synchronization of coupled harmonic oscillators in a dynamic proximity network, Automatica 45(10)(2009)2286-2291.

[27] H. Zhang, J. Zhou, Z. Liu, Synchronization of networked harmonic oscillators with communication delays under local instantaneous interaction, Journal of Dynamic Systems, Measurement, and Control 134(6)(2012)061009.

[28] W. Sun, J. Lü, S. Chen, X. Yu, Synchronisation of directed coupled harmonic oscillators with sampled-data, IET Control Theory \& Applications 8(11)(2014)937-947.

[29] Q. Song, W. Yu, J. Cao, F. Liu, Reaching synchronization in networked harmonic oscillators with outdated position data, IEEE Transactions on Cybernetics (2015), http://dx.doi.org/10.1109/TCYB.2015.2451651, in press.

[30] S. I. Niculescu, Delay Effects on Stability: A Robust Control Approach, Springer, 2001.

[31] K. Gu, V. L. Kharitonov, J. Chen, Stability of Time-Delay Systems, Springer, 2003.

[32] S. Ruan, J. Wei, On the zeros of transcendental functions with applications to stability of delay differential equations with two delays, Dynamics of Continuous, Discrete and Impulsive Systems, Series A: Mathematical Analysis 10(2003)863-874.

[33] W. Ren, On consensus algorithms for double-integrator dynamics, IEEE Transactions on Automatic Control 53(6)(2008)1503-1509.

[34] Y. Hong, G. Chen, L. Bushnell, Distributed observers design for leader-following control of multiagent networks, Automatica 44(3)(2008)846-850.

[35] A. Abdessameud, A. Tayebi, On consensus algorithms for double-integrator dynamics without velocity measurements and with input constraints, Systems \& Control Letters 59(12)(2010)812-821.

[36] J. Mei, W. Ren, G. Ma, Distributed coordination for second-order multi-agent systems with nonlinear dynamics using only relative position measurements, Automatica 49(5)(2013)1419-1427.

[37] Y. Zhang, Y. Yang, Y. Zhao, Finite-time consensus tracking for harmonic oscillators using both state feedback control and output feedback control, International Journal of Robust and Nonlinear Control 23(8)(2013)878-893.

[38] R. A. Horn, C. R. Johnson, Topics in Matrix Analysis, Cambridge University Press, 1991.

[39] D. G. Luenberger, An introduction to observers, IEEE Transactions on Automatic Control 16(6)(1971)596-602.

[40] P. C. Parks, V. Hahn, Stability Theory, Prentice-Hall, 1993. 
Chenglin Wen received the bachelor's and masters degrees in mathematics from Henan University, Kaifeng, China, and Zhengzhou University, Zhengzhou, China, and the Ph.D. degree from Northwestern Polytechnical University, Xi'an, China, in 1986, 1996, and 1999, respectively. He is now a Chair Professor in the College of Electrical Engineering, Henan University of Technology, Zhengzhou, China. His current research interests include multi-sensor networked information fusion theory, multitarget tracking, fault diagnosis of complex systems and devices, reliability assessment and health control, recognition, and tracking of hypersonic vehicle. He is a Committee Member of Intelligent Automation Committee and Process Fault Diagnosis and Security Committee of Chinese Association of Automation.

Fang Liu received the B.Eng. and M.Sc. degrees from the Department of Electrical Engineering, Xi'an Jiaotong University, Xi'an, China, in 1994 and 1997, respectively, and the Ph.D. degree from the Department of Electrical and Computer Engineering McMaster University, Hamilton, Ontario, Canada in 2006. From 1997 to 2000, she was with Shandong Electric Power Corporation. In 2001, she worked as a research assistant in the Department of Electrical and Computer Engineering at University of Western Ontario, London, Canada. Dr. Liu is now an Associate Professor and the Associate Dean in the School of Information Engineering, Huanghuai University, Zhumadian, Henan, China. Her research interests include control theory and applications, complex networks, electrical apparatus and data fusion. 
Qiang Song received the Master degree from the Department of Electrical Engineering, Xi'an Jiaotong University, Xi'an, China in 1995 and the Ph.D. degree from the School of Automation, Southeast University, Nanjing, China in 2011. He is now with the College of Electrical Engineering, Henan University of Technology, Zhengzhou, China. From December 2013 to December 2014, he was a Postdoctoral Research Fellow at the Department of Mechanical Engineering, The University of Hong Kong, Hong Kong. Dr. Song has published over ten peer-reviewed international journal papers, including two highly cited Essential Science Indicators (ESI) papers on pinning control of networked systems. Dr. Song is an Active Reviewer for several international journals and was selected as an Outstanding Reviewer for IET Control Theory \& Applications in 2014. His current research interests include control theory and applications, complex networks and multi-agent systems.

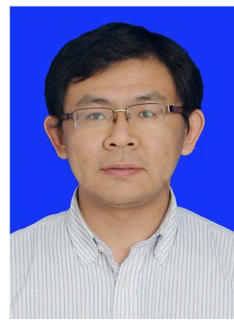

Xiaoliang Feng received the Master degree from the School of Automation, Hangzhou Dianzi University, Hangzhou, China in 2010 and the Ph.D. degree from the College of computer and information engineering, Hohai University, Nanjing, China in 2013. He is now with the College of Electrical Engineering, Henan University of Technology, Zhengzhou, China, and his current research interests include networked control system, and information fusion. 